\title{
Permutational powers of a graph
}

\author{
Matteo Cavaleri Daniele D'Angeli Alfredo Donno \\ Università Niccolò Cusano \\ Rome, Italy \\ \{matteo.cavaleri, daniele.dangeli, alfredo.donno\}@unicusano.it
}

Submitted: Nov 25, 2018; Accepted: Oct 7, 2019; Published: Oct 25, 2019

(C) The authors. Released under the CC BY-ND license (International 4.0).

\begin{abstract}
This paper introduces a new graph construction, the permutational power of a graph, whose adjacency matrix is obtained by the composition of a permutation matrix with the adjacency matrix of the graph. It is shown that this construction recovers the classical zig-zag product of graphs when the permutation is an involution, but is in fact more general. We start by discussing necessary and sufficient conditions on the permutation and on the adjacency matrix of a graph to guarantee their composition to represent an adjacency matrix of a graph, then we focus our attention on the cases in which the permutational power does not reduce to a zigzag product. We show that the cases of interest are those in which the adjacency matrix is singular. This leads us to frame our problem in the context of equitable partitions, obtained by identifying vertices having the same neighborhood. The families of cyclic and complete bipartite graphs are treated in details.
\end{abstract}

Mathematics Subject Classifications: 05C50, 05C76, 05C78

\section{Introduction}

Graphs are among the most popular and useful tools used in Mathematics to model aspects of real life. Their relatively simple and natural definition makes these objects very versatile and adaptable in many areas of mathematical research. Usually graphs are studied via their adjacency matrix, and it is an interesting task to investigate the relationship between the geometrical properties of a graph and the algebraic properties of the corresponding adjacency matrix. Moreover, there exists a correspondence between operations that can be performed on graphs and operations on matrices. See, for example, [5, 7, 11, 12, 16]. In the last years, a very intriguing product of graphs that has been introduced is the so called zig-zag product: iterated applications of this product allow to construct infinite families of expanders.

The zig-zag product of two graphs was introduced in [15] as a construction allowing to 
produce, starting from a large graph $G$ and a small graph $H$, a new graph $G(2) H$ which inherits the size from the large one, the degree from the small one, and the expansion property from both the graphs. In [15], it is explicitly described how iteration of this construction, together with the standard squaring, provides an infinite family of constantdegree expander graphs, starting from a particular graph representing the building block of this construction (see [13] for further details on expanders). Topological properties of the zig-zag product have been studied in the paper [6]. It is worth mentioning that the zig-zag product has also interesting connections with Geometric group theory, as it is true that the zig-zag product of the Cayley graphs of two groups returns the Cayley graph of the semi-direct product of the groups [1]. See also the book [3] for connections of the zig-zag product with other graph compositions and random walks.

In the present paper, we show that one can in any case replace the role of $G$ by an appropriate permutation matrix $P$ of order 2 . In other words, if $G$ has $k$ vertices, one can realize the adjacency matrix of the zig-zag product $G(\mathrm{Z}) H$ as the product $\tilde{A}_{H} P \tilde{A}_{H}$, where $A_{H}$ is the adjacency matrix of the graph $H$ (so that $\tilde{A}_{H}=I_{k} \otimes A_{H}$ can be regarded as the adjacency matrix of the graph obtained by taking $k \geqslant 1$ disjoint copies of $H$ ), and $P$ is a symmetric permutation matrix. Keeping this in mind, one can ask if it is possible to get the adjacency matrix of some graph (i.e., a symmetric $\tilde{A}_{H} P \tilde{A}_{H}$ ) by considering also permutation matrices $P$ that are not symmetric. The permutational power graph corresponds exactly to this case. Now it is clear that, whenever we have $\tilde{A}_{H} P \tilde{A}_{H}$ symmetric (and so it can interpreted as the adjacency matrix of an undirected graph) we can ask if the same graph can be obtained via the "classical" zig-zag product (by using a symmetric $P$ ). This problem is strictly related to the singularity of the matrix $A_{H}$. In the case in which $A_{H}$ is invertible, there is no chance to get a symmetric $\tilde{A}_{H} P \tilde{A}_{H}$ with a nonsymmetric $P$. For this reason, we focus our attention on the cases where $A_{H}$ is singular.

In fact, the reason why the graph $H$ is singular says a lot about the nature of its permutational power. The most general case is when we only know that the adjacency matrix of $H$ is singular. We consider this case in Section 3.1, proving that a permutation $p$ induces a permutational power of $H$ if its projection on the range of the adjacency matrix of $H$ is symmetric. Moreover, a permutational power of $H$ with respect to $p$ can be obtained by a classical zig-zag product if there exists an involution $q$ sharing with $p$ the same projection on the range of the adjacency matrix of $H$.

One of the reasons why the adjacency matrix of $H$ may be singular is that there are vertices sharing the same neighborhood: this is one of the cases analyzed in Section 4 via the notion of neighborhood equitable partition. Actually, in the framework of random matrices and random graphs, it is conjectured that this is the generic reason why $H$ could be singular (for the symmetric case see [4]). The graph obtained collapsing the equivalent vertices together (in a precise way), can be singular or not. In the latter case every permutational power of $H$ can be obtained via the classical zig-zag product (Theorem 27): the easiest examples are the complete bipartite graphs. On the other extreme, the adjacency matrix of $H$ can be singular even if there are no vertices sharing the same neighborhood: the easiest examples are cyclic graphs $C_{n}$ with $n$ divisible by 4 . 
In this paper we show, among other results, that:

- the symmetry of $\tilde{A}_{H} P \tilde{A}_{H}$ only depends on the symmetry of the projection of $P$ on the range of the matrix $\tilde{A}_{H}$ (see Theorem 9 and Corollary 10);

- there are permutational powers that do not appear as classical zig-zag products (see Corollary 14);

- we can restrict our attention, in a specific sense, to graphs whose adjacency matrix is singular with pairwise distinct rows (see Corollary 21, Corollary 22 and Theorem 27).

Moreover, the cases in which $H$ is a cycle or a complete bipartite graph are studied in details (see Sections 3.2 and 3.3, and Section 4.1).

\section{Preliminaries and motivations}

In this paper we will denote by $G=\left(V_{G}, E_{G}\right)$ a finite undirected graph with vertex set $V_{G}$ and edge set $E_{G}$. If $\{u, v\} \in E_{G}$, we say that the vertices $u$ and $v$ are adjacent in $G$, and we write $u \sim v$. Observe that loops and multi-edges are allowed. A path of length $t$ in $G$ is a sequence $u_{0}, u_{1}, \ldots, u_{t}$ of vertices such that $u_{i} \sim u_{i+1}$. The graph is connected if, for every $u, v \in V_{G}$, there exists a path $u_{0}, u_{1}, \ldots, u_{t}$ in $G$ such that $u_{0}=u$ and $u_{t}=v$.

Suppose $\left|V_{G}\right|=n$. We denote by $A_{G}=\left(a_{u, v}\right)_{u, v \in V_{G}}$ the adjacency matrix of $G$, i.e., the square matrix of size $n$ whose entry $a_{u, v}$ is equal to the number of edges joining $u$ and $v$. The degree of a vertex $u \in V_{G}$ is defined as $\operatorname{deg}(u)=\sum_{v \in V_{G}} a_{u, v}$. In particular, we say that $G$ is regular of degree $d$, or $d$-regular, if $\operatorname{deg}(u)=d$, for each $u \in V_{G}$. For such a graph $G$, the normalized adjacency matrix is defined as $A_{G}^{\prime}=\frac{1}{d} A_{G}$.

We recall now the definition of the zig-zag product of two graphs. This is a noncommutative graph product producing a graph whose degree depends only on the degree of the second component graph, and providing large and sparse graphs. We need some notation.

Let $G=\left(V_{G}, E_{G}\right)$ be a $d$-regular graph over $n$ vertices. Suppose that we have a set of $d$ colors (labels), that we identify with the set $[d]=\{1,2, \ldots, d\}$. We can assume that, for each vertex $v \in V_{G}$, the edges incident to $v$ are labelled by a color $h \in[d]$ near $v$, and that any two distinct edges issuing from $v$ have a different color near $v$. This allows to define the rotation map $\operatorname{Rot}_{G}: V_{G} \times[d] \longrightarrow V_{G} \times[d]$ such that, for all $v \in V_{G}$ and $h \in[d]$,

$$
\operatorname{Rot}_{G}(v, h)=(w, k)
$$

if there exists an edge joining $v$ and $w$ in $G$, which is colored by the color $h$ near $v$ and by the color $k$ near $w$. Note that it may be $h \neq k$. Moreover, the composition $\operatorname{Rot}_{G} \circ \operatorname{Rot}_{G}$ is the identity map.

Definition 1. Let $G=\left(V_{G}, E_{G}\right)$ be a $d_{G^{-}}$-regular graph, with $\left|V_{G}\right|=n$, and let $H=$ $\left(V_{H}, E_{H}\right)$ be a $d_{H}$-regular graph such that $\left|V_{H}\right|=d_{G}$. Let $\operatorname{Rot}_{G}\left(\operatorname{resp} \operatorname{Rot}_{H}\right)$ denote the 
rotation map of $G$ (resp. $H$ ). The zig-zag product $G(2) H$ is the regular graph of degree $d_{H}^{2}$ with vertex set $V_{G} \times V_{H}$, identified with the set $V_{G} \times\left[d_{G}\right]$, and whose edges are described by the rotation map

$$
\operatorname{Rot}_{G(\mathbb{Z})}((v, k),(i, j))=\left((w, l),\left(j^{\prime}, i^{\prime}\right)\right),
$$

for all $v \in V_{G}, k \in\left[d_{G}\right], i, j \in\left[d_{H}\right]$, if:

1. $\operatorname{Rot}_{H}(k, i)=\left(k^{\prime}, i^{\prime}\right)$,

2. $\operatorname{Rot}_{G}\left(v, k^{\prime}\right)=\left(w, l^{\prime}\right)$,

3. $\operatorname{Rot}_{H}\left(l^{\prime}, j\right)=\left(l, j^{\prime}\right)$,

where $w \in V_{G}, l, k^{\prime}, l^{\prime} \in\left[d_{G}\right]$ and $i^{\prime}, j^{\prime} \in\left[d_{H}\right]$.

Notice that when $(v, k)$ is the first argument of the map $\operatorname{Rot}_{G(\mathbb{Z}) H}$, it must be interpreted as a vertex of the product $G(2) H$, but when it is the argument of the map $\operatorname{Rot}_{G}$, it must be interpreted as the edge of $G$ with origin in $v \in V_{G}$ and initial color $k$. Moreover, observe that labels in $G(2) H$ are elements from $\left[d_{H}\right]^{2}$. The vertex set of $G(2) H$ is partitioned into $n$ clouds, indexed by the vertices of $G$, where by definition the $v$-cloud, with $v \in V_{G}$, is constituted by the vertices $(v, 1),(v, 2), \ldots,\left(v, d_{G}\right)$. Two vertices $(v, k)$ and $(w, l)$ of $G(2) H$ are adjacent in $G(2) H$ if it is possible to go from $(v, k)$ to $(w, l)$ by a sequence of three steps of the following form:

1. a first step "zig" within the initial cloud, from the vertex $(v, k)$ to the vertex $\left(v, k^{\prime}\right)$, described by $\operatorname{Rot}_{H}(k, i)=\left(k^{\prime}, i^{\prime}\right)$;

2. a second step jumping from the $v$-cloud to the $w$-cloud, from the vertex $\left(v, k^{\prime}\right)$ to the vertex $\left(w, l^{\prime}\right)$, described by $\operatorname{Rot}_{G}\left(v, k^{\prime}\right)=\left(w, l^{\prime}\right)$;

3. a third step "zag" within the new cloud, from the vertex $\left(w, l^{\prime}\right)$ to the vertex $(w, l)$, described by $\operatorname{Rot}_{H}\left(l^{\prime}, j\right)=\left(l, j^{\prime}\right)$.

Example 2. Consider the graphs $G$ and $H$ in Fig. 1.
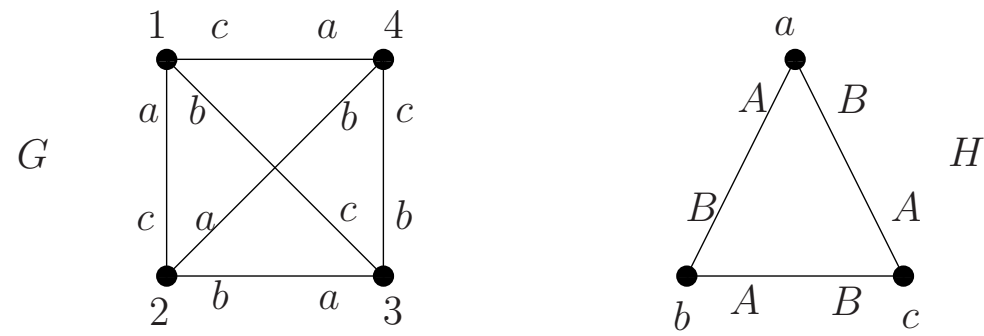

Figure 1: The graphs $G$ and $H$ of Example 2.

It follows from Definition 1 that the graph $G(2) H$, which is depicted in Fig. 2, is the graph with vertex set $\{1,2,3,4\} \times\{a, b, c\}$, whose edges are labelled by ordered pairs $(i, j)$ 
from $\{A, B\}^{2}$. If we ask, for instance, which are the vertices adjacent to $(1, a)$ in $G(2) H$, we have to take into account that:

$$
\begin{aligned}
\operatorname{Rot}_{H}(a, A) & =(b, B) \\
\operatorname{Rot}_{G}(1, b) & =(3, c) \quad \operatorname{Rot}_{H}(a, B)=(c, A) \\
\operatorname{Rot}_{H}(c, A)=(a, B) & \operatorname{Rot}_{H}(c, B)=(b, A) \\
\operatorname{Rot}_{H}(a, A) & =(b, B) \quad \operatorname{Rot}_{H}(a, B)=(c, A) .
\end{aligned}
$$

In this way, we conclude that the vertex $(1, a)$ in $G(2) H$ is adjacent to the vertices $(3, a),(3, b),(4, b),(4, c)$.

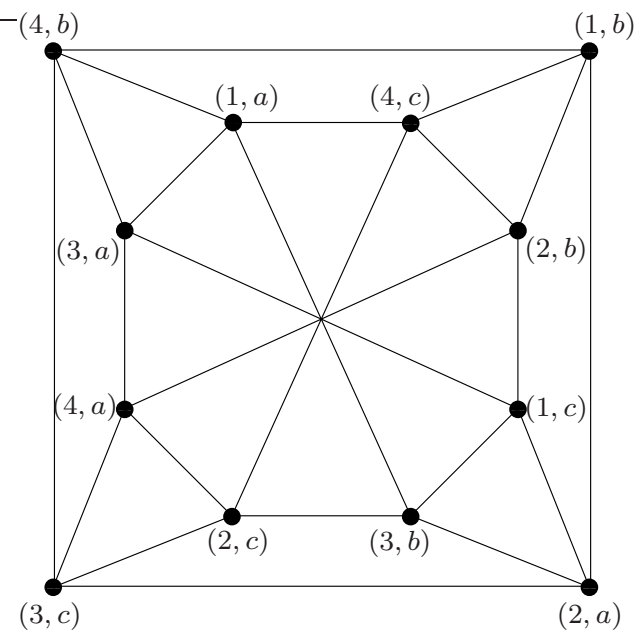

Figure 2: The graph $G(\mathrm{C}) H$ of Example 2.

Let us analyze the adjacency matrix of $G(2) H$. Let $A_{G}$ (resp. $A_{H}$ ) be the adjacency matrix of the graph $G$ (resp. $H$ ). Let $I_{n}$ denote the identity matrix of size $n$, for each $n \geqslant 1$. It follows from the definition of zig-zag product that the adjacency matrix of $G(2) H$ is $A_{G}$ (2) $H=\left(I_{\left|V_{G}\right|} \otimes A_{H}\right) P_{G}\left(I_{\left|V_{G}\right|} \otimes A_{H}\right)$ (see [15]), where $P_{G}$ is the permutation matrix of size $\left|V_{G}\right|\left|V_{H}\right|$ associated with the $\operatorname{map} \operatorname{Rot}_{G}$, i.e.,

$$
P_{G(v, k),(w, l)}= \begin{cases}1 & \text { if } v \sim w \text { in } G \text { by an edge labelled } k \text { near } v \text { and } l \text { near } w \\ 0 & \text { otherwise. }\end{cases}
$$

If $G$ is the graph of Example 2, we have 


$$
P_{G}=\left(\begin{array}{ccc|ccc|ccc|ccc}
0 & 0 & 0 & 0 & 0 & 1 & 0 & 0 & 0 & 0 & 0 & 0 \\
0 & 0 & 0 & 0 & 0 & 0 & 0 & 0 & 1 & 0 & 0 & 0 \\
0 & 0 & 0 & 0 & 0 & 0 & 0 & 0 & 0 & 1 & 0 & 0 \\
\hline 0 & 0 & 0 & 0 & 0 & 0 & 0 & 0 & 0 & 0 & 1 & 0 \\
0 & 0 & 0 & 0 & 0 & 0 & 1 & 0 & 0 & 0 & 0 & 0 \\
1 & 0 & 0 & 0 & 0 & 0 & 0 & 0 & 0 & 0 & 0 & 0 \\
\hline 0 & 0 & 0 & 0 & 1 & 0 & 0 & 0 & 0 & 0 & 0 & 0 \\
0 & 0 & 0 & 0 & 0 & 0 & 0 & 0 & 0 & 0 & 0 & 1 \\
0 & 1 & 0 & 0 & 0 & 0 & 0 & 0 & 0 & 0 & 0 & 0 \\
\hline 0 & 0 & 1 & 0 & 0 & 0 & 0 & 0 & 0 & 0 & 0 & 0 \\
0 & 0 & 0 & 1 & 0 & 0 & 0 & 0 & 0 & 0 & 0 & 0 \\
0 & 0 & 0 & 0 & 0 & 0 & 0 & 1 & 0 & 0 & 0 & 0
\end{array}\right) .
$$

Observe that $P_{G}^{2}=I_{12}$, as the rotation map is an involution. Equivalently, $P_{G}$ is a symmetric matrix.

For each positive integer $k$, put $\tilde{A}_{H}=I_{k} \otimes A_{H}$ (observe that, for $k=1$, one has $\tilde{A}_{H}=A_{H}$ ). Our paper is motivated by the following observation: if we are given a graph $H$, and we consider $k$ copies of such graph, and we are also given a symmetric permutation matrix $P$ of size $k\left|V_{H}\right|$ (i.e., a matrix corresponding to a permutation of order 2 of $k\left|V_{H}\right|$ elements), then the symmetric matrix $M=\tilde{A}_{H} P \tilde{A}_{H}$ can be regarded as the adjacency matrix of a graph composition of type "zig-zag", where the jump steps are codified by the matrix permutation $P$. In other words, the first factor graph is not essential to perform the zig-zag construction, one just needs the permutation matrix $P$ describing the rotation map.

\section{Permutational powers of a graph}

The concluding remark of Section 2 can be formalized as follows. For every $n \geqslant 1$, let $\operatorname{Sym}(n)$ denote the symmetric group on $n$ elements. Take a regular graph $H$ on $m$ vertices, and fix a positive integer $k \geqslant 1$. Let $P$ be a symmetric matrix permutation on $k m$ elements, that is, the permutation $p \in \operatorname{Sym}(\mathrm{km})$ associated with $P$ is the composition of disjoint transpositions (with possibly some fixed elements). Let us identify $V_{H}$ with the set $\{0,1, \ldots, m-1\}$, and similarly identify the $k m$ vertices obtained by taking $k$ copies of $H$ with the set $\{0,1, \ldots, k m-1\}$. Observe that each number $x \in\{0,1, \ldots, k m-1\}$ admits a unique representation as

$$
x=i m+j, \quad \text { with } i=0,1, \ldots, k-1 \text { and } j=0,1, \ldots, m-1 .
$$

With this interpretation, we can think that the vertex $x$ is the $j$-th vertex belonging to the $i$-th copy of the graph $H$.

Let us construct now a labelled $m$-regular graph $G$ on $k$ vertices as follows. Vertices will be named $0,1, \ldots, k-1$, whereas edges will have labels (colors) $0,1, \ldots, m-1$ around each vertex. More precisely, if the transposition $\tau=(s t)$ appears in $p$, with $s=i_{s} m+j_{s}$ 
and $t=i_{t} m+j_{t}$, then we connect the vertices $i_{s}$ and $i_{t}$ in $G$ by an edge labelled $j_{s}$ near the vertex $i_{s}$ and by $j_{t}$ near the vertex $i_{t}$. If $u=i_{u} m+j_{u}$ is an element fixed by $p$, there will be a loop at the vertex $i_{u}$ with two labels equal to $j_{u}$. In this situation, the graph with adjacency matrix $\tilde{A}_{H} P \tilde{A}_{H}$ coincides with the graph $G(2) H$, where $G$ has been constructed as described above.

Example 3. Consider 3 copies of the cyclic graph $C_{4}$ on 4 vertices, with vertex set $\{0,1,2,3\}$, and the following permutation of order 2 :

$$
p=\left(\begin{array}{ll}
0 & 11
\end{array}\right)\left(\begin{array}{l}
1 \\
9
\end{array}\right)(25)(36)(410)(78)
$$

on 12 elements, and let $P$ be the associated permutation matrix. Then the matrix $\left(I_{3} \otimes\right.$ $\left.A_{C_{4}}\right) P\left(I_{3} \otimes A_{C_{4}}\right)$ is symmetric, and it is nothing but the adjacency matrix of the zig-zag product $G(2) C_{4}$, where $G$ is the labelled graph depicted in Fig. 3.
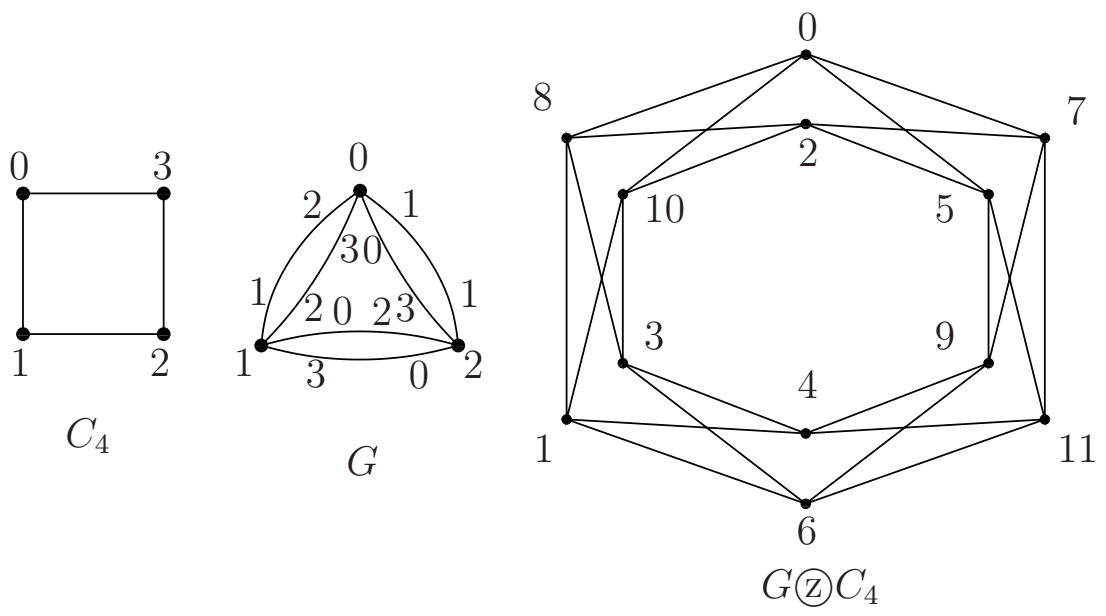

Figure 3: The graph $G(2) C_{4}$ of Example 3.

Remark 4. Even if the zig-zag product has been defined in [15] for regular graphs, in order to construct increasing sequences of regular expander graphs, our construction shows that one can also start from a nonregular graph $H$. In fact, if one takes $k$ copies of $H$ and a permutation matrix on $k\left|V_{H}\right|$ elements such that the product $M=\tilde{A}_{H} P \tilde{A}_{H}$ is symmetric, then $M$ can be regarded as the adjacency matrix of a graph obtained from $H$ by a composition of type "zig-zag".

Example 5. In Fig. 5, two copies of a nonregular graph $H$ on 6 vertices, denoted $H_{0}$

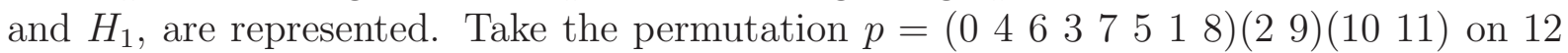
elements. Construct the graph $G$ associated with $p$ (see Fig. 4). The graph resulting from the construction described above appears in the bottom of Fig. 5. Notice that it is not a regular graph. If, for instance, we ask which are the vertices in $G(2) H$ which are adjacent to the vertex 2 , we have to think that 2 is a vertex belonging to the copy indexed by 0 , and its neighbors are the vertices $0,1,3$. Such vertices are mapped by $p$, respectively, to 


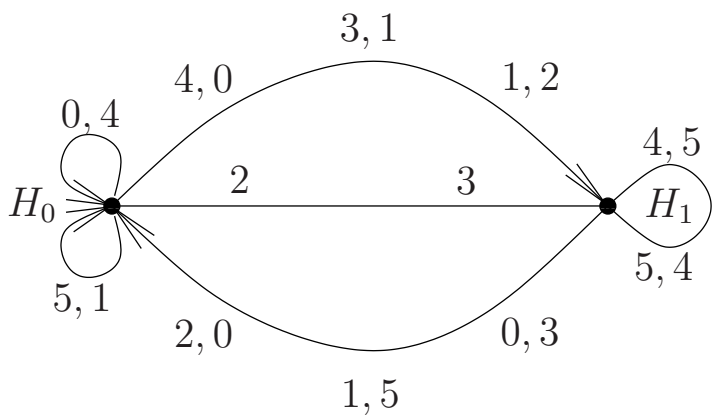

Figure 4: The graph $G$ of Example 5 .

the vertices $4,8,7$. Now, the only neighbor of 4 in the copy $H_{0}$ is 3 ; the neighbor of 7 in $H_{1}$ is 8 ; finally, the neighbors of 8 in $H_{1}$ are the three vertices $6,7,9$. We then conclude that the vertices adjacent to 2 are exactly the vertices $3,8,6,7,9$.

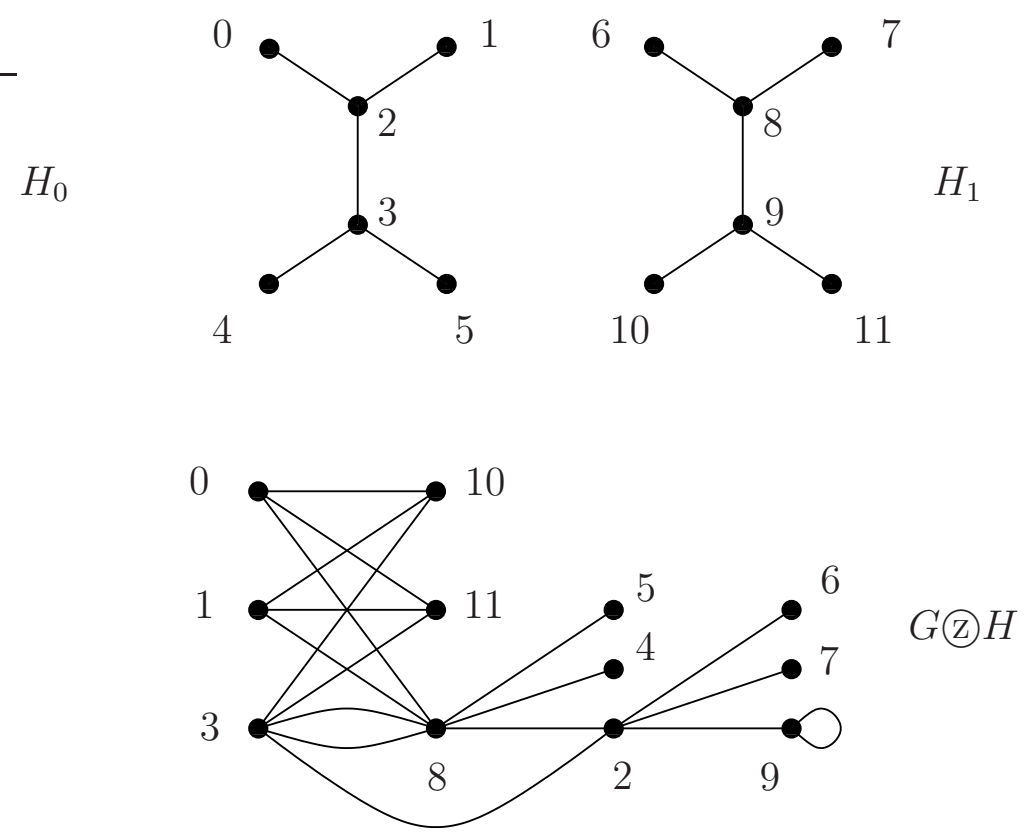

Figure 5: The graphs $H_{0}, H_{1}, G(2) H$ of Example 5.

Notice that in Example 3 the graph $G$ constructed starting from the permutation is undirected, whereas it is directed in Example 5, due to the fact that the order of the permutation is not 2. In Fig. 4 the labels in the arc directed from the copy 0 to the copy 1 must be interpreted as follows: 4, 0 corresponds to the fact that $p(4)=6 \cdot 1+0=6$; 3,1 to the fact that $p(3)=6 \cdot 1+1=7$ and 1,2 to the fact that $p(1)=6 \cdot 1+2=8$. Similarly, the loop at the 0 copy starting with 5 and ending with 1 corresponds to the fact $p(5)=6 \cdot 0+1=1$. The undirected edge and the undirected loops correspond to the transpositions (2 9) and (10 11), respectively. 
One can also ask what happens when the matrix $\tilde{A}_{H} P \tilde{A}_{H}$ is not symmetric: in this situation, the resulting matrix can be regarded as the signed adjacency matrix of a directed graph, what leads to the possibility of defining a zig-zag product of directed graphs, containing the classical product as a particular case. This general situation will not be investigated in the present paper.

On the other hand, it may happen that, even if the permutation $p$ is not of order 2 , anyway one has directed edges from each vertex in the neighborhood of $v$ to each vertex in the neighborhood of $w$ and viceversa, producing an undirected graph. This is the case we are interested in.

Definition 6. Let $H=\left(V_{H}, E_{H}\right)$ be a graph with adjacency matrix $A_{H}$, and let $p \in$ $\operatorname{Sym}\left(k\left|V_{H}\right|\right)$, with $k \geqslant 1$, with associated permutation matrix $P$. Let $\tilde{A}_{H}=I_{k} \otimes A_{H}$. If $M=\tilde{A}_{H} P \tilde{A}_{H}$ is symmetric, the graph whose adjacency matrix is $M$ is the permutational $k$-th power of $H$ with respect to $p$.

The main questions that we address in our paper are the following.

1. Given a graph $H$, with adjacency matrix $A_{H}$, and taken a positive integer $k$, is it possible to find a nonsymmetric permutation matrix $P$ on $k\left|V_{H}\right|$ elements such that the matrix $\tilde{A}_{H} P \tilde{A}_{H}$ is symmetric?

2. If this is the case, under which conditions does there exist a symmetric permutation matrix $Q$ such that $\tilde{A}_{H} P \tilde{A}_{H}=\tilde{A}_{H} Q \tilde{A}_{H}$ ? In other words, when can such a permutational power of $H$ be obtained by the classical zig-zag product?

As an example, observe that the resulting graph in Fig. 3 can also be obtained by choosing the permutation $p^{\prime}=\left(\begin{array}{lllllllllll}0 & 11 & 3 & 6 & 10 & 7 & 8 & 4 & 1 & 9 & 2\end{array}\right)$ on 12 elements. However, we will see that there exist permutational powers of graphs which cannot be obtained by the classical zig-zag construction of Definition 1 (see Corollary 14).

\subsection{An algebraic interpretation}

Let us start an algebraic investigation of the symmetry condition

$$
\left(\tilde{A}_{H} P \tilde{A}_{H}\right)^{T}=\tilde{A}_{H} P \tilde{A}_{H} .
$$

Lemma 7. If the adjacency matrix $A_{H}$ of the graph $H$ is invertible, then any permutational power of $H$ is a zig-zag product.

Proof. Observe that the matrix $\tilde{A}_{H}$ is invertible if and only if the matrix $A_{H}$ is invertible. Moreover, the matrix $\tilde{A}_{H}$ is symmetric, as $A_{H}$ is symmetric. This implies that

$$
\left(\tilde{A}_{H} P \tilde{A}_{H}\right)^{T}=\tilde{A}_{H} P \tilde{A}_{H} \quad \Longleftrightarrow \quad P^{T}=P
$$

and so the graph with adjacency matrix $\tilde{A}_{H} P \tilde{A}_{H}$ is a zig-zag construction.

By virtue of Lemma 7, the interesting cases that it is worth investigating are given by graphs $H$ whose adjacency matrix is singular. Examples of such graphs are [2]: 
- the cyclic graph $C_{n}$ on $n=4 h$ vertices;

- the path graph $P_{n}$ on $n=2 h+1$ vertices;

- all bipartite graphs with an odd number of vertices;

- graphs with two or more vertices sharing the same neighborhood (e.g., the complete bipartite graph $\left.K_{m, n}\right)$.

Put $M=\tilde{A}_{H} P \tilde{A}_{H}$, with $\left|V_{H}\right|=m$. Let $x, y \in\{0,1, \ldots, k m-1\}$, with representation $x=i_{x} m+j_{x} ; y=i_{y} m+j_{y}$, with $i_{x}, i_{y} \in\{0,1, \ldots, k-1\}$ and $j_{x}, j_{y} \in\{0,1, \ldots, m-1\}$. Then we have:

$$
\begin{aligned}
M_{x, y} & =\sum_{h, l=0}^{k m-1} \widetilde{a}_{x, l} p_{l, h} \widetilde{a}_{h, y} \\
& =\#\left\{j_{l} \sim j_{x}: p\left(i_{x} m+j_{l}\right)=i_{y} m+j_{l^{\prime}}, j_{l^{\prime}} \sim j_{y}\right\} \\
& =\#\left\{j_{l} \sim j_{x}: \exists j_{l^{\prime}} \sim j_{y}: p\left(i_{x} m+j_{l}\right)=i_{y} m+j_{l^{\prime}}\right\} .
\end{aligned}
$$

If we repeat the same computation for the entry $M_{y, x}$, we deduce that Eq. (1) is satisfied if and only if, for each $i_{x}, i_{y} \in\{0,1, \ldots, k-1\}$ and $j_{x}, j_{y} \in\{0,1, \ldots, m-1\}$, one has:

$\#\left\{j_{l} \sim j_{x}: \exists j_{l^{\prime}} \sim j_{y}: p\left(i_{x} m+j_{l}\right)=i_{y} m+j_{l^{\prime}}\right\}=\#\left\{j_{l^{\prime}} \sim j_{y}: \exists j_{l} \sim j_{x}: p\left(i_{y} m+j_{l^{\prime}}\right)=i_{x} m+j_{l}\right\}$.

In other words, the number of the neighbors $j_{l}$ of the vertex $j_{x}$ in the copy $i_{x}$ of $H$ such that $p$ maps $i_{x} m+j_{l}$ to $i_{y} m+j_{l^{\prime}}$, where $j_{l^{\prime}}$ is a neighbor of the vertex $j_{y}$ in the copy $i_{y}$, must be equal to the number of the neighbors $j_{l^{\prime}}$ of the vertex $j_{y}$ in the copy $i_{y}$ of $H$ such that $p$ maps $i_{y} m+j_{l^{\prime}}$ to $i_{x} m+j_{l}$, where $j_{l}$ is a neighbor of the vertex $j_{x}$ in the copy $i_{x}$.

By a similar argument, one can prove the following proposition.

Proposition 8. Let $H$ be a graph. Let $k$ be a positive integer, and suppose that $P$ is a permutation matrix of size $k\left|V_{H}\right|$ such that the matrix $\tilde{A}_{H} P \tilde{A}_{H}$ is symmetric. Then also the matrix $\tilde{A}_{H} P^{-1} \tilde{A}_{H}$ is symmetric.

The same argument does not apply to the whole cyclic group generated by the permutation $P$, as there exist explicit examples showing that, if the matrix $\tilde{A}_{H} P \tilde{A}_{H}$ is symmetric, then the matrix $\tilde{A}_{H} P^{h} \tilde{A}_{H}$ need not be symmetric for any integer $h$.

The matrix $A_{H}$ is symmetric, so that it admits all real eigenvalues, and an orthonormal basis of eigenvectors. Moreover, since we are dealing with a singular matrix, we can assume that 0 is an eigenvalue for $A_{H}$. Put $\lambda_{0}=0$ and let $\lambda_{1}, \ldots, \lambda_{s}$ be the nonzero eigenvalues of $A_{H}$. Let $m_{i}$ be the multiplicity of $\lambda_{i}$, for each $i=0,1, \ldots, s$, and let us denote by $E_{i}$ the corresponding eigenspace, so that $E_{0}=\operatorname{ker} A_{H}$.

Now, the spectrum of the matrix $\tilde{A}_{H}$ coincides with the spectrum of $A_{H}$, but the eigenvalue $\lambda_{i}$ has multiplicity $k m_{i}$, for each $i=0,1, \ldots, s$. The corresponding eigenspace is $\widetilde{E}_{i}=\mathbb{R}^{k} \otimes E_{i}$. Notice that, due to the symmetry of $A_{H}$, we have

$$
\mathbb{R}^{m}=\bigoplus_{i=0}^{s} E_{i}=\operatorname{ker} A_{H} \oplus\left(\bigoplus_{i=1}^{s} E_{i}\right)
$$


where the eigenspaces $E_{i}$ are pairwise orthogonal. Since

$$
\tilde{A}_{H} P \tilde{A}_{H}=\tilde{A}_{H} P^{T} \tilde{A}_{H} \Longleftrightarrow \tilde{A}_{H}\left(P-P^{T}\right) \tilde{A}_{H}=O,
$$

Eq. (1) is satisfied if and only if the matrix $P-P^{T}$ maps the space $\bigoplus_{i=1}^{s} \widetilde{E}_{i}$ to the space $\widetilde{E}_{0}=\mathbb{R}^{k} \otimes \operatorname{ker} A_{H}$.

Similarly, given a permutation matrix $P$ such that $\tilde{A}_{H} P \tilde{A}_{H}$ is symmetric, and whose order is not 2 , a permutation matrix $Q$ satisfies $\tilde{A}_{H} P \tilde{A}_{H}=\tilde{A}_{H} Q \tilde{A}_{H}$ if and only if the matrix $P-Q$ maps the space $\bigoplus_{i=1}^{s} \widetilde{E}_{i}$ to the space $\widetilde{E}_{0}=\mathbb{R}^{k} \otimes \operatorname{ker} A_{H}$.

Taking an orthonormal basis from each eigenspace $\widetilde{E}_{i}$, we construct an orthogonal matrix $U$ such that $U^{T} \tilde{A}_{H} U$ is diagonal. Put

$$
U=\left(U_{1} \mid U_{0}\right)
$$

where $U_{1}$ is the submatrix whose columns form a basis of the space $\bigoplus_{i=1}^{s} \widetilde{E}_{i}$, which is orthogonal to $\widetilde{E}_{0}$, and $U_{0}$ is the submatrix whose columns form a basis of $\widetilde{E}_{0}$.

Theorem 9. For any two permutation matrices $P$ and $Q$ we have

$$
\tilde{A}_{H} P \tilde{A}_{H}=\tilde{A}_{H} Q \tilde{A}_{H} \Longleftrightarrow U_{1}^{T} P U_{1}=U_{1}^{T} Q U_{1} .
$$

Proof. We have $\tilde{A}_{H} P \tilde{A}_{H}=\tilde{A}_{H} Q \tilde{A}_{H}$ if and only if the matrix $P-Q$ maps the space $\bigoplus_{i=1}^{s} \widetilde{E}_{i}$ to the space $\widetilde{E}_{0}=\mathbb{R}^{k} \otimes \operatorname{ker} A_{H}$. This is equivalent to ask that, for every two columns $u, v$ in $U_{1}$, one has that $(P-Q) u$ is orthogonal to $v$. Therefore, it must be $U_{1}^{T}(P-Q) U_{1}=O$, that is the claim.

Corollary 10. The matrix $\tilde{A}_{H} P \tilde{A}_{H}$ is symmetric if and only if $U_{1}^{T} P U_{1}$ is symmetric.

Proof. We can apply Theorem 9 to the case $Q=P^{T}$.

From an algebraic point of view, Theorem 9 and Corollary 10 give the complete answers to our two main questions, and we are going to apply them, in the next subsections, to the case of cyclic graphs. In Section 4, we will be interested in finding more geometric conditions, that in particular cases (e.g., complete bipartite graphs) characterize the permutations $p$ inducing a permutational $k$-th power.

\subsection{Cyclic graphs}

In this section we are going to completely characterize the permutational 1-st powers of the cyclic graph $C_{n}$. The adjacency matrix of $C_{n}$ is the circulant matrix

$$
A_{C_{n}}=\left(\begin{array}{cccccc}
0 & 1 & 0 & \cdots & 0 & 1 \\
1 & 0 & 1 & & & 0 \\
0 & 1 & \ddots & \ddots & & \vdots \\
\vdots & & \ddots & \ddots & \ddots & 0 \\
0 & & & \ddots & \ddots & 1 \\
1 & 0 & \cdots & 0 & 1 & 0
\end{array}\right) .
$$


Let us denote with $R_{n}$ the set of complex $n$-th roots of 1 . It is a classical fact [2] that, for every $\lambda \in R_{n}$, the vector $v_{\lambda}=\left(\lambda, \lambda^{2}, \ldots, \lambda^{n-1}, 1\right)$ is eigenvector for $A_{C_{n}}$ of eigenvalue $\lambda+\bar{\lambda}$.

Consider a permutation $p \in \operatorname{Sym}(n)$ and the associated permutation matrix $P$. We are going to investigate under which conditions the matrix $A_{C_{n}} P A_{C_{n}}$ is symmetric. As we already noticed, if $n$ is not divisible by 4 , the matrix $A_{C_{n}}$ is invertible and so by Lemma 7 the matrix $P$ must be symmetric. From now on, assume that $n=4 k$. In the set $[n]$ we define an involution $i \mapsto i^{*}$, where $i^{*}$ is the element such that $\left|i-i^{*}\right|=2 k$. Since $n$ is even, we have $-\lambda \in R_{n}$ and $\lambda^{i^{*}}=-\lambda^{i}$.

Lemma 11. Let $\zeta$ be an $n$-th primitive root of 1 , and let $i_{1}, i_{2}, i_{3}, i_{4} \in[n]$. Then:

$$
\zeta^{i_{1}}+\zeta^{i_{2}}=\zeta^{i_{3}}+\zeta^{i_{4}} \Longrightarrow\left(i_{1}=i_{2}^{*} \wedge i_{3}=i_{4}^{*}\right) \vee\left\{i_{1}, i_{2}\right\}=\left\{i_{3}, i_{4}\right\} .
$$

Proof. Set $\lambda_{1}=\zeta^{i_{1}}, \lambda_{2}=\zeta^{i_{2}}, \lambda_{3}=\zeta^{i_{3}^{*}}, \lambda_{4}=\zeta^{i_{4}^{*}}$, then we have $\lambda_{1}+\lambda_{2}+\lambda_{3}+\lambda_{4}=0$, with $\lambda_{1}, \lambda_{2}, \lambda_{3}, \lambda_{4}$ roots of unity. As a consequence of Theorem 6 in [14], the only possibility is that $\left(\lambda_{1}=-\lambda_{2} \wedge \lambda_{3}=-\lambda_{4}\right) \vee\left(\lambda_{1}=-\lambda_{3} \wedge \lambda_{2}=-\lambda_{4}\right) \vee\left(\lambda_{1}=-\lambda_{4} \wedge \lambda_{2}=-\lambda_{3}\right)$. Since $\zeta$ is primitive, for $i, j \in[n]$, we have $\zeta^{i}=\zeta^{j} \Longrightarrow i=j$ : the thesis follows.

In the spirit of the introductory remarks to Theorem 9 , the matrix $A_{C_{n}} P A_{C_{n}}$ is symmetric if and only if $\left(P-P^{T}\right) v_{\lambda} \in \operatorname{ker} A_{C_{n}}$, for each $\lambda \in R_{n} \backslash\{ \pm i\}$, since the vectors $\left\{v_{\lambda}: \lambda \in R_{n}, \lambda \neq \pm i\right\}$ are a basis for the range of $A_{C_{n}}$. Now we have:

$$
\left(P-P^{T}\right) v_{\lambda}=\left(\begin{array}{c}
\lambda^{p(1)}-\lambda^{p^{-1}(1)} \\
\lambda^{p(2)}-\lambda^{p^{-1}(2)} \\
\vdots \\
\lambda^{p(n-1)}-\lambda^{p^{-1}(n-1)} \\
\lambda^{p(n)}-\lambda^{p^{-1}(n)}
\end{array}\right)=\left(\begin{array}{c}
\lambda^{p(1)}+\lambda^{p^{-1}(1)^{*}} \\
\lambda^{p(2)}+\lambda^{p^{-1}(2)^{*}} \\
\vdots \\
\lambda^{p(n-1)}+\lambda^{p^{-1}(n-1)^{*}} \\
\lambda^{p(n)}+\lambda^{p^{-1}(n)^{*}}
\end{array}\right) .
$$

The condition $\left(P-P^{T}\right) v_{\lambda} \in \operatorname{ker} A_{C_{n}}$ is equivalent to:

$$
\left\{\begin{array}{l}
\lambda^{p(2)}+\lambda^{p^{-1}(2)^{*}}+\lambda^{p(n)}+\lambda^{p^{-1}(n)^{*}}=0 \\
\lambda^{p(1)}+\lambda^{p^{-1}(1)^{*}}+\lambda^{p(3)}+\lambda^{p^{-1}(3)^{*}}=0 \\
\vdots \\
\lambda^{p(2 i)}+\lambda^{p^{-1}(2 i)^{*}}+\lambda^{p(2 i+2)}+\lambda^{p^{-1}(2 i+2)^{*}}=0 \\
\lambda^{p(2 i-1)}+\lambda^{p^{-1}(2 i-1)^{*}}+\lambda^{p(2 i+1)}+\lambda^{p^{-1}(2 i+1)^{*}}=0 \\
\vdots \\
\lambda^{p(n-2)}+\lambda^{p^{-1}(n-2)^{*}}+\lambda^{p(n)}+\lambda^{p^{-1}(n)^{*}}=0 \\
\lambda^{p(1)}+\lambda^{p^{-1}(1)^{*}}+\lambda^{p(n-1)}+\lambda^{p^{-1}(n-1)^{*}}=0 .
\end{array}\right.
$$

By suitably coupling these equations, we get

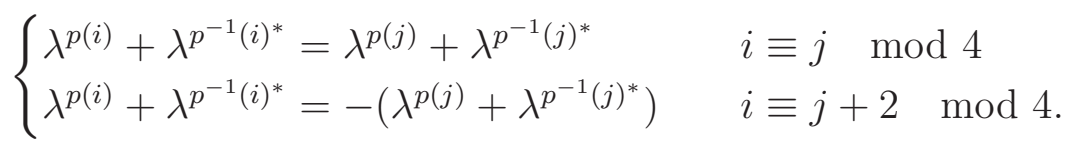


If $\zeta \in R_{n}$ is primitive, by virtue of Lemma 11, the condition $\left(P-P^{T}\right) v_{\zeta} \in \operatorname{ker} A_{C_{n}}$ is equivalent to:

$$
\begin{aligned}
& (p(i), p(j))=\left(p^{-1}(i), p^{-1}(j)\right) \text { or } \\
& (p(i), p(j))=\left(p^{-1}(j)^{*}, p^{-1}(i)^{*}\right)
\end{aligned}
$$

for $i \equiv j \bmod 4$;

$$
\begin{aligned}
(p(i), p(j)) & =\left(p^{-1}(i), p^{-1}(j)\right) \text { or } \\
(p(i), p(j)) & =\left(p^{-1}(j), p^{-1}(i)\right) \text { or } \\
\left(p(i), p^{-1}(i)\right) & =\left(p(j)^{*}, p^{-1}(j)^{*}\right)
\end{aligned}
$$

for $i \equiv j+2 \bmod 4$. This is true because, for $i \neq j$, the condition $\left\{p(i), p^{-1}(i)^{*}\right\}=$ $\left\{p(j), p^{-1}(j)^{*}\right\}$ of Lemma 11 implies $p(i)=p^{-1}(j)^{*}$ and $p(j)=p^{-1}(i)^{*}$.

We are now in position to prove the following theorem.

Theorem 12. If $n>8$,

$$
A_{C_{n}} P A_{C_{n}}=\left(A_{C_{n}} P A_{C_{n}}\right)^{T} \Longleftrightarrow P=P^{T}
$$

In other words, there is no permutational 1-st power of $C_{n}$ with respect to a nonsymmetric permutation.

Proof. By contradiction, suppose $P \neq P^{T}$. Without loss of generality $p(1) \neq p^{-1}(1)$. By Eq. (2) we have $p(1)=p^{-1}(5)^{*}$ and $p(1)=p^{-1}(9)^{*}$ : it implies $p^{-1}(5)=p^{-1}(9)$, that is impossible.

Observe that the graph $C_{4}$ belongs to the class of complete bipartite graphs, that we are going to analyze in detail in Section 4.1. The remaining case is the cycle graph $C_{8}$, which is studied in the next section.

\subsection{The cycle $C_{8}$}

In order to obtain necessary conditions for the symmetry of the matrix $A_{C_{8}} P A_{C_{8}}$, we study the behavior of the permutation $p^{2}$. From the first line of Eq. (2) and the first line of Eq. (3) we have that if $i \equiv j \bmod 2$ then

$$
p^{2}(i)=i \Longleftrightarrow p^{2}(j)=j .
$$

Then if $A_{C_{8}} P A_{C_{8}}$ is symmetric, we only have the following 4 possibilities:

a) $\left\{i \in[8]: p^{2}(i)=i\right\}=[8]$

b) $\left\{i \in[8]: p^{2}(i)=i\right\}=\{2,4,6,8\}$

c) $\left\{i \in[8]: p^{2}(i)=i\right\}=\{1,3,5,7\}$ 
d) $\left\{i \in[8]: p^{2}(i)=i\right\}=\emptyset$.

The case a) concerns permutations of order 2 and then the classical zig-zag product. In the case b), the even numbers are in 1-cycles or 2-cycles and there is a 4-cycle containing the odd numbers. We know that $p(1) \neq p^{-1}(1)$. Considering $i=1$ and $j=3$ in Eq. (3) we get $p(1)=p^{-1}(3)$ or $p(1)=p(3)^{*}$. On the other hand, Eq. (2) with $i=3$ and $j=7$ gives $p(3)^{*}=p^{-1}(7)$, so that it must be $p^{2}(1)$ equal to 3 or 7 . By an analogous argument we have:

$$
\left\{p^{2}(1), p^{2}(5)\right\}=\{3,7\} \text { and }\left\{p^{2}(3), p^{2}(7)\right\}=\{1,5\} \text {. }
$$

As a consequence, the only 4-cycles that could appear in the case b) are:

$$
\sigma_{1}=(1375), \sigma_{2}=(1735), \sigma_{3}=(1537), \sigma_{4}=(1573) \text {. }
$$

By an explicit computation (our conditions, a priori, are only necessary) one can check that $A_{C_{8}} P A_{C_{8}}$ is symmetric when $p=\sigma_{i}$ (and therefore also when $p$ is a product of $\sigma_{i}$ with a permutation of order 2 of the even numbers).

An analogous argument in the case c) gives

$$
\left\{p^{2}(2), p^{2}(6)\right\}=\{4,8\} \text { and }\left\{p^{2}(4), p^{2}(8)\right\}=\{2,6\}
$$

and the 4-cycles

$$
\tau_{1}=(2486), \tau_{2}=(2846), \tau_{3}=(2648), \tau_{4}=(2684) .
$$

So we have that $p$ is a permutation of case c) if and only if $p$ is the product of a $\tau_{i}$ with a permutation of order 2 of the odd numbers.

In the case d) we can apply Eq. (2) to all pairs $\left(i, i^{*}\right)$, obtaining

$$
p(i)=\left(p^{-1}\left(i^{*}\right)\right)^{*}, \quad \forall i \in[n] ;
$$

that is, $p$ is conjugated to its inverse by the permutation induced by the involution *. Moreover, we have that Eq. (4) and Eq. (5) hold. In particular $p^{4}$ should be the identity or an involution and therefore $p$ is an 8-cycle or the product of two 4-cycles. Moreover, if $p$ were a 8-cycle, we would have that $p^{4}(i)=i^{*}$ for any $i \in[n]$, that is $p=\left(a b c d a^{*} b^{*} c^{*} d^{*}\right)$ for some $a, b, c, d \in[n]$. By applying Eq. (6) to $p$ we have $\left(a b c d a^{*} b^{*} c^{*} d^{*}\right)=\left(d c b a d^{*} c^{*} b^{*} a^{*}\right)$, hence this is impossible. Thus $p$ is a product of two disjoint 4-cycles. If the permutation sends even (resp. odd) numbers in even (resp. odd) numbers, we are just in the case $p=\tau_{i} \sigma_{j}$. If this is not the case, the cycles alternate odd with even numbers; by Eq. (4) and Eq. (5) such a permutation must be of the form:

$$
p=(1 a b c)(5 d e f),
$$

with $a, c, d, f$ even, $\{b, e\}=\{3,7\}, a^{*} \neq c$ and $d^{*} \neq f$. Finally, applying Eq. (6) for $i=1,5, a$, we have that $f=a^{*}, d=c^{*}$ and $e=b^{*}$, and therefore the permutation $p$ should be of the form $p=(1 a b c)\left(5 c^{*} b^{*} a^{*}\right)$, where we can freely choose $b \in\{3,7\}$, 
$a \in\{2,4,6,8\}$ and $c$ between the two even numbers different from $a^{*}$.

The corresponding 16 permutations are:

$$
\begin{array}{llll}
\gamma_{1}=(1234)(5876) & \gamma_{2}=(1436)(5278) & \gamma_{3}=(1638)(5472) & \gamma_{4}=(1832)(5674) \\
\gamma_{5}=(1274)(5836) & \gamma_{6}=(1476)(5238) & \gamma_{7}=(1678)(5432) & \gamma_{8}=(1872)(5634) \\
\gamma_{9}=(1238)(5476) & \gamma_{10}=(1432)(5678) & \gamma_{11}=(1634)(5472) & \gamma_{12}=(1836)(5274) \\
\gamma_{13}=(1278)(5436) & \gamma_{14}=(1472)(5638) & \gamma_{15}=(1674)(5832) & \gamma_{16}=(1876)(5234)
\end{array}
$$

By a direct computation, one can check that all these permutations make $A_{C_{8}} P A_{C_{8}}$ symmetric.

Summarizing, the permutations $p \in \operatorname{Sym}(8)$ such that $p^{2} \neq I d$ and $A_{C_{8}} P A_{C_{8}}$ is symmetric are the following 112 permutations:

$$
q \sigma_{i}, \quad s \tau_{j}, \quad \sigma_{i} \tau_{j}, \quad \gamma_{k}
$$

where $i, j=1, \ldots, 4, k=1, \ldots, 16$, and $q$ (resp. $s$ ) is a permutation of order at most 2 fixing each odd (resp. even) number.

Example 13. There exist permutational 1-st powers of $C_{8}$ that can be obtained by a permutation of order 2 and there exist permutational 1-st powers of $C_{8}$ for which this is impossible. For instance, consider the permutation $p_{1}=\tau_{4}=(2684)$ with associated matrix $P_{1}$, and suppose $q_{1} \in \operatorname{Sym}(8)$ is such that $A_{C_{8}} Q_{1} A_{C_{8}}=A_{C_{8}} P_{1} A_{C_{8}}$. The analogue of Eq. (2) for $p_{1}$ and $q_{1}$ gives $\left(q_{1}(2), q_{1}(6)\right)=(6,8)$ or $\left(q_{1}(2), q_{1}(6)\right)=\left(8^{*}, 6^{*}\right)=(4,2)$. In both cases $q_{1}^{2} \neq I d$. The graph with adjacency matrix $A_{C_{8}} P_{1} A_{C_{8}}$ is depicted in Fig. 6 (observe that it consists of two connected components).

On the other hand, let $p_{2}=\gamma_{1}=(1234)(5876)$, with associated matrix $P_{2}$. Then one can check that, by defining $q_{2}=(12)(34)(58)(76)$, one has $A_{C_{8}} P_{2} A_{C_{8}}=A_{C_{8}} Q_{2} A_{C_{8}}$, showing that both the possibilities may occur.

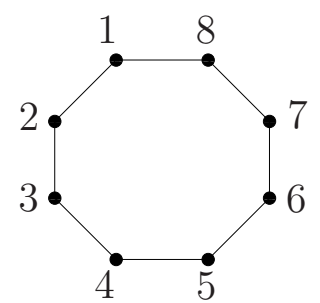

$C_{8}$
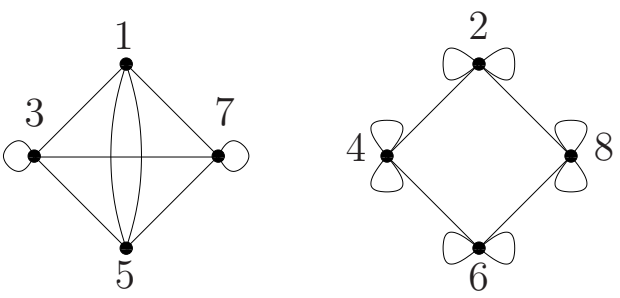

Figure 6: The graph $C_{8}$ and its permutation 1-st power with respect to $p_{1}$.

Corollary 14. There exist permutational powers that cannot be expressed as zig-zag products. 


\section{Equitable partitions}

In this section we will use the notion of equitable partition. The main idea is that, whenever we call vertices that have the same neighborhoods equivalent, then we obtain a so-called equitable partition. This observation allows us to deeply explore the structure of the permutations giving rise to permutational powers and, in particular, to detect those permutational powers that actually can be obtained by a classical zig-zag product.

Equitable partitions have a number of significant applications in Graph Theory: for example, the vertex set partition of a graph under the action of a group of automorphisms is always equitable. This fact has been used in the context of graph isomorphism algorithms (we refer to the book [9] for more details).

Let $G=\left(V_{G}, E_{G}\right)$ be a graph, with $\left|V_{G}\right|=n$. With a given partition $\pi=\left\{C_{1}, \ldots, C_{m}\right\}$ of $V_{G}$ we can associate an $n \times m$ matrix $M_{\pi}$, called the characteristic matrix of $\pi$, defined as follows. For each $v \in V_{G}$, and $i \in\{1, \ldots, m\}$, one has

$$
\left(M_{\pi}\right)_{v, i}= \begin{cases}\frac{1}{\sqrt{\left|C_{i}\right|}} & \text { if } v \in C_{i} \\ 0 & \text { otherwise. }\end{cases}
$$

It is easy to check that:

$$
M_{\pi}^{T} M_{\pi}=I_{m}
$$

Moreover we can use the characteristic matrix of $\pi$ to define the $m \times m$ matrix $A_{G} / \pi=$ $M_{\pi}^{T} A_{G} M_{\pi}$. This matrix represents the restriction of the matrix $A_{G}$ to the parts of $\pi$.

Definition 15. [17] For each vertex $v \in V_{G}$, put $B_{1}(v)=\left\{u \in V_{G}: v \sim u\right\}$, that is, $B_{1}(v)$ is the neighborhood of $v$ in $G$. Then a partition $\pi$ of $V_{G}$ is equitable if, for all $i \in\{1, \ldots, m\}$, and all $v, w \in C_{i}$, one has $\left|B_{1}(v) \cap C_{j}\right|=\left|B_{1}(w) \cap C_{j}\right|$, for each $j \in\{1, \ldots, m\}$.

It is known that the equitability condition of Definition 15 is equivalent to each of the following (the reader can refer to $[8,9,10]$ ):

- $A_{G} M_{\pi}=M_{\pi}\left(A_{G} / \pi\right)$;

- $M_{\pi}^{T} A_{G}=\left(A_{G} / \pi\right) M_{\pi}^{T}$;

- $A_{G} M_{\pi} M_{\pi}^{T}=M_{\pi} M_{\pi}^{T} A_{G}$.

Moreover, if $\pi$ is equitable, one has

$$
\left(M_{\pi}^{T} A_{G} M_{\pi}\right)_{i, j}=\sum_{h, l=1}^{n} m_{h, i} a_{h, l} m_{l, j}=\sum_{h \sim l} m_{h, i} m_{l, j},
$$

so that the entry $\left(A_{G} / \pi\right)_{i, j}$ equals the total number of edges connecting a vertex of $C_{i}$ to a vertex of $C_{j}$, multiplied by $\frac{1}{\sqrt{\left|C_{i}\right|\left|C_{j}\right|}}$. In other words, the matrix $A_{G} / \pi$ can be regarded, up 
to a suitable normalization, as the adjacency matrix of the quotient graph $G / \pi$ obtained from $G$ by taking the quotient of $V_{G}$ modulo the equivalence relation defined by $\pi$.

In what follows we will mostly focus on a very specific equitable partition $\hat{\pi}$, the one induced by the relation "to have the same neighborhood". This partition is natural in the context of graphs and fits into our setting of permutational powers of $G$. It concretely corresponds to the existence of two or more rows in the matrix $A_{G}$ which are equal. This condition assures that $A_{G}$ is not invertible and this is exactly the case we are interested in, by virtue of Lemma 7 .

Definition 16. Let $G=\left(V_{G}, E_{G}\right)$. The partition $\hat{\pi}$ is the partition of $V_{G}$ such that $v, w \in V_{G}$ are in the same part if $B_{1}(v)=B_{1}(w)$.

By definition, $\hat{\pi}$ is an equitable partition; we will call it the neighborhood partition of $V_{G}$. The following proposition shows that $\hat{\pi}$ has an even stronger property.

Proposition 17. Let $\hat{\pi}$ be the neighborhood partition of $V_{G}$. Then

$$
A_{G} M_{\hat{\pi}} M_{\hat{\pi}}^{T}=A_{G}=M_{\hat{\pi}} M_{\hat{\pi}}^{T} A_{G} .
$$

Proof. For all $u, v \in V_{G}$, we have:

$$
\left(M_{\hat{\pi}} M_{\hat{\pi}}^{T}\right)_{u, v}= \begin{cases}\frac{1}{\left|C_{u, v}\right|} & \text { if } u \text { and } v \text { belong to the same class } C_{u, v} \\ 0 & \text { otherwise. }\end{cases}
$$

This implies:

$$
\begin{aligned}
\left(M_{\hat{\pi}} M_{\hat{\pi}}^{T} A_{G}\right)_{u, v} & =\sum_{w \in V_{G}}\left(M_{\hat{\pi}} M_{\hat{\pi}}^{T}\right)_{u, w} a_{w, v}=\sum_{w \in C_{u, w}} a_{w, v} \frac{1}{\left|C_{u, w}\right|}=\sum_{w \in C_{u, w}} a_{u, v} \frac{1}{\left|C_{u, w}\right|} \\
& =a_{u, v} \frac{\left|C_{u, w}\right|}{\left|C_{u, w}\right|}=\left(A_{G}\right)_{u, v},
\end{aligned}
$$

where we have used that $a_{w, v}=a_{u, v}$, because $w$ and $u$ are in the same part of $\hat{\pi}$. Since $\hat{\pi}$ is equitable, we conclude that $A_{G} M_{\hat{\pi}} M_{\hat{\pi}}^{T}=M_{\hat{\pi}} M_{\hat{\pi}}^{T} A_{G}=A_{G}$.

In particular $G / \hat{\pi}$ is the graph where the vertices of $G$ with the same neighborhood are identified.

Example 18. Let $H$ be the graph on 6 vertices, with $V_{H}=\{0,1,2,3,4,5\}$, of Example 5 . We have $\hat{\pi}=\left\{C_{1}, C_{2}, C_{3}, C_{4}\right\}$, where $C_{1}=\{0,1\}, C_{2}=\{2\}, C_{3}=\{3\}$, and $C_{4}=\{4,5\}$. The adjacency matrix of $H$ and the characteristic matrix of $\hat{\pi}$ are, respectively:

$$
A_{H}=\left(\begin{array}{cccccc}
0 & 0 & 1 & 0 & 0 & 0 \\
0 & 0 & 1 & 0 & 0 & 0 \\
1 & 1 & 0 & 1 & 0 & 0 \\
0 & 0 & 1 & 0 & 1 & 1 \\
0 & 0 & 0 & 1 & 0 & 0 \\
0 & 0 & 0 & 1 & 0 & 0
\end{array}\right) \quad M_{\hat{\pi}}=\left(\begin{array}{cccc}
1 / \sqrt{2} & 0 & 0 & 0 \\
1 / \sqrt{2} & 0 & 0 & 0 \\
0 & 1 & 0 & 0 \\
0 & 0 & 1 & 0 \\
0 & 0 & 0 & 1 / \sqrt{2} \\
0 & 0 & 0 & 1 / \sqrt{2}
\end{array}\right) .
$$


A direct computation gives

$$
M_{\hat{\pi}}^{T} A_{H} M_{\hat{\pi}}=\left(\begin{array}{cccc}
0 & \sqrt{2} & 0 & 0 \\
\sqrt{2} & 0 & 1 & 0 \\
0 & 1 & 0 & \sqrt{2} \\
0 & 0 & \sqrt{2} & 0
\end{array}\right),
$$

which is, up to normalization, the adjacency matrix of the quotient graph $H / \hat{\pi}$ in Fig. 7 .

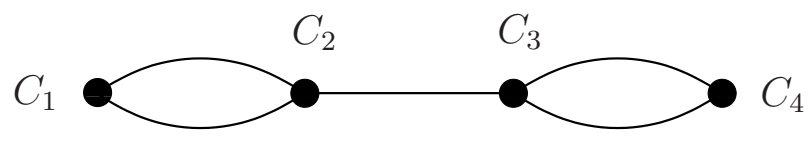

Figure 7: The quotient graph $H / \hat{\pi}$ of Example 18.

We focus now our attention on the case of a graph $H$ for which we want to investigate permutational $k$-th powers. Observe that the neighborhood partition $\hat{\pi}$ can be considered also for the graph obtained by taking $k$ disjoint copies of $H$, and it is induced in a very natural way by the neighborhood partition of the vertex set $V_{H}$. As usual, we put $\tilde{A}_{H}=I_{k} \otimes A_{H}$, and $P$ is a matrix permutation acting on $k\left|V_{H}\right|$ elements, with $k \geqslant 1$.

Proposition 19. Let $H=\left(V_{H}, E_{H}\right)$ be a graph with adjacency matrix $A_{H}$. Then vertices having the same neighborhood in $H$ produce ( $k$ copies of) vertices having the same neighborhood in any permutational $k$-th power of $H$.

Proof. Put $\left|V_{H}\right|=n$. Let $A_{H}=\left(a_{i, j}\right)_{i, j=0, \ldots, n-1}$ be the adjacency matrix of $H$ and assume that $a_{r, j}=a_{s, j}$ for each $j=0, \ldots, n-1$, that is, the $r$-th and the $s$-th vertex of $H$ have the same neighborhood in $H$. Consider the permutational $k$-th power of $H$ induced by a permutation $p$, with associated permutation matrix $P$, and enumerate its vertices as $0,1, \ldots, k n-1$. Then, for each $h=0, \ldots, k-1$ and $j=0, \ldots, k n-1$, we have:

$$
\begin{aligned}
\left(\tilde{A}_{H} P \tilde{A}_{H}\right)_{h n+r, j} & =\sum_{l, m=0}^{k n-1}\left(\tilde{A}_{H}\right)_{h n+r, l} P_{l, m}\left(\tilde{A}_{H}\right)_{m, j} \\
& =\sum_{l=0}^{k n-1}\left(\tilde{A}_{H}\right)_{h n+r, l}\left(\tilde{A}_{H}\right)_{p(l), j} \\
& =\sum_{x_{l}=0}^{k-1} \sum_{y_{l}=0}^{n-1}\left(\tilde{A}_{H}\right)_{h n+r, x_{l} n+y_{l}}\left(\tilde{A}_{H}\right)_{p\left(x_{l} n+y_{l}\right), j} \\
& =\sum_{y_{l}=0}^{n-1} a_{r, y_{l}}\left(\tilde{A}_{H}\right)_{p\left(h n+y_{l}\right), j},
\end{aligned}
$$


and similarly

$$
\left(\tilde{A}_{H} P \tilde{A}_{H}\right)_{h n+s, j}=\sum_{y_{l}=0}^{n-1} a_{s, y_{l}}\left(\tilde{A}_{H}\right)_{p\left(h n+y_{l}\right), j} .
$$

Since $a_{r, y_{l}}=a_{s, y_{l}}$ by hypothesis, we get the claim.

In Example 5 (Fig. 5), observe that the vertices 0 and 1, and the vertices 4 and 5, have the same neighborhood in the graph $H$ : this implies that the pairs of vertices 0 and $1 ; 4$ and 5; 6 and 7; 10 and 11, have the same neighborhood in the permutational power of $H$.

On the other hand, the condition of Proposition 19 is only sufficient, as it may occur that in the final graph two vertices share the same neighborhood, but the same property does not hold in the original graph. In Fig. 8, we have represented the cyclic graph $C_{8}$,

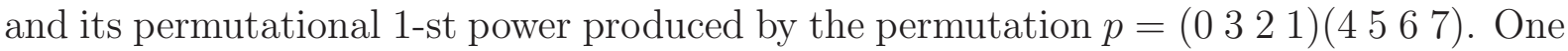
can directly check that the vertices 0 and 4 have the same neighborhood in the final graph, as well as the vertices 3 and 7 ; however, the equitable partition given by the neighborhood in $C_{8}$ is trivial, because there are no vertices sharing the same neighborhood in $C_{8}$.
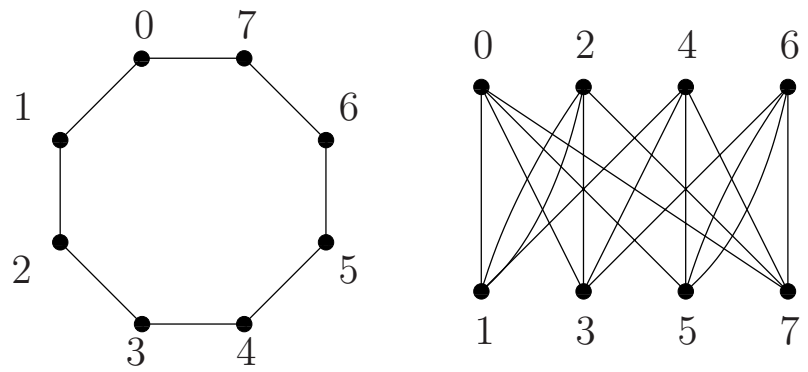

Figure 8: The cyclic graph $C_{8}$ and a permutational 1-st power.

Given a matrix permutation $P$ and the partition $\hat{\pi}$, we define $P / \hat{\pi}=M_{\hat{\pi}}^{T} P M_{\hat{\pi}}$ in order to describe the action of the permutation on the classes induced by $\hat{\pi}$. We have the following reduction result.

Theorem 20. Let $A_{H}$ be the adjacency matrix of a graph $H$. Then

$$
\tilde{A}_{H} P_{1} \tilde{A}_{H}=\tilde{A}_{H} P_{2} \tilde{A}_{H} \Longleftrightarrow\left(\tilde{A}_{H} / \hat{\pi}\right)\left(P_{1} / \hat{\pi}\right)\left(\tilde{A}_{H} / \hat{\pi}\right)=\left(\tilde{A}_{H} / \hat{\pi}\right)\left(P_{2} / \hat{\pi}\right)\left(\tilde{A}_{H} / \hat{\pi}\right) .
$$

Proof. By Eq. (8),

$$
\tilde{A}_{H} P_{i} \tilde{A}_{H}=\tilde{A}_{H} M_{\hat{\pi}} M_{\hat{\pi}}^{T} P_{i} M_{\hat{\pi}} M_{\hat{\pi}}^{T} \tilde{A}_{H}
$$

by definition of $P_{i} / \hat{\pi}$ :

$$
=\tilde{A}_{H} M_{\hat{\pi}}\left(P_{i} / \hat{\pi}\right) M_{\hat{\pi}}^{T} \tilde{A}_{H}
$$

by equitability properties:

$$
=M_{\hat{\pi}}\left(\tilde{A}_{H} / \hat{\pi}\right)\left(P_{i} / \hat{\pi}\right)\left(\tilde{A}_{H} / \hat{\pi}\right) M_{\hat{\pi}}^{T},
$$


that is:

$$
\tilde{A}_{H} P_{i} \tilde{A}_{H}=M_{\hat{\pi}}\left(\tilde{A}_{H} / \hat{\pi}\right)\left(P_{i} / \hat{\pi}\right)\left(\tilde{A}_{H} / \hat{\pi}\right) M_{\hat{\pi}}^{T}
$$

and then

$$
\left(\tilde{A}_{H} / \hat{\pi}\right)\left(P_{1} / \hat{\pi}\right)\left(\tilde{A}_{H} / \hat{\pi}\right)=\left(\tilde{A}_{H} / \hat{\pi}\right)\left(P_{2} / \hat{\pi}\right)\left(\tilde{A}_{H} / \hat{\pi}\right) \Longrightarrow \tilde{A}_{H} P_{1} \tilde{A}_{H}=\tilde{A}_{H} P_{2} \tilde{A}_{H} .
$$

On the other hand, by Eq. (7),

$$
\left(\tilde{A}_{H} / \hat{\pi}\right)\left(P_{i} / \hat{\pi}\right)\left(\tilde{A}_{H} / \hat{\pi}\right)=M_{\hat{\pi}}^{T} M_{\hat{\pi}}\left(\tilde{A}_{H} / \hat{\pi}\right)\left(P_{i} / \hat{\pi}\right)\left(\tilde{A}_{H} / \hat{\pi}\right) M_{\hat{\pi}}^{T} M_{\hat{\pi}}
$$

and by Eq. (9)

$$
=M_{\hat{\pi}}^{T} \tilde{A}_{H} P_{i} \tilde{A}_{H} M_{\hat{\pi}}
$$

Therefore

$$
\left(\tilde{A}_{H} / \hat{\pi}\right)\left(P_{i} / \hat{\pi}\right)\left(\tilde{A}_{H} / \hat{\pi}\right)=M_{\hat{\pi}}^{T} \tilde{A}_{H} P_{i} \tilde{A}_{H} M_{\hat{\pi}}
$$

and then

$$
\tilde{A}_{H} P_{1} \tilde{A}_{H}=\tilde{A}_{H} P_{2} \tilde{A}_{H} \Longrightarrow\left(\tilde{A}_{H} / \hat{\pi}\right)\left(P_{1} / \hat{\pi}\right)\left(\tilde{A}_{H} / \hat{\pi}\right)=\left(\tilde{A}_{H} / \hat{\pi}\right)\left(P_{2} / \hat{\pi}\right)\left(\tilde{A}_{H} / \hat{\pi}\right) .
$$

Corollary 21. Let $A_{H}$ be the adjacency matrix of a graph $H$. Then

$$
\tilde{A}_{H} P \tilde{A}_{H} \text { is symmetric } \Longleftrightarrow\left(\tilde{A}_{H} / \hat{\pi}\right)(P / \hat{\pi})\left(\tilde{A}_{H} / \hat{\pi}\right) \text { is symmetric. }
$$

Proof. It is a particular case of Theorem 20 when $P_{1}=P$ and $P_{2}=P^{T}$.

Corollary 22. If $P_{1} / \hat{\pi}=P_{2} / \hat{\pi}$ then $\tilde{A}_{H} P_{1} \tilde{A}_{H}=\tilde{A}_{H} P_{2} \tilde{A}_{H}$. In particular, if $P / \hat{\pi}$ is symmetric then $\tilde{A}_{H} P \tilde{A}_{H}$ is symmetric.

Proof. If $P_{1} / \hat{\pi}=P_{2} / \hat{\pi}$ then $\left(\tilde{A}_{H} / \hat{\pi}\right)\left(P_{1} / \hat{\pi}\right)\left(\tilde{A}_{H} / \hat{\pi}\right)=\left(\tilde{A}_{H} / \hat{\pi}\right)\left(P_{2} / \hat{\pi}\right)\left(\tilde{A}_{H} / \hat{\pi}\right)$, and by virtue of Theorem 20 the first claim is proved. The second claim easily follows from Corollary 21.

The following proposition answers our Question (2) in Section 2, in the context of the neighborhood partition $\hat{\pi}$.

Proposition 23. If $P / \hat{\pi}$ is symmetric then there exists $Q$ of order 2 such that $\tilde{A}_{H} P \tilde{A}_{H}=$ $\tilde{A}_{H} Q \tilde{A}_{H}$.

Proof. Let $\hat{\pi}=\left\{C_{1}, \ldots, C_{m}\right\}$ be the neighborhood partition on the vertex set of $k$ disjoint copies of $H$, naturally induced by the neighborhood partition of $V_{H}$. Notice that, for a fixed $i$, the entry $(P / \hat{\pi})_{i, j}$ counts, up to the factor $1 / \sqrt{\left|C_{i}\right|\left|C_{j}\right|}$, the number of elements in the class $C_{i}$ moved by the permutation $p$ to the class $C_{j}$. We define

$$
V_{i, j}^{(p)}=\left\{v \in C_{i}: p(v) \in C_{j}\right\}
$$


for any $i, j \in\{1, \ldots, m\}$. Therefore $(P / \hat{\pi})_{i, j}=(P / \hat{\pi})_{j, i}$ if and only if $\left|V_{i, j}^{(p)}\right|=\left|V_{j, i}^{(p)}\right|$ for any $i, j$. Moreover for every $i$ there exists a partition $C_{i}=\cup_{j=1}^{m} V_{i, j}^{(p)}$. Observe that some $V_{i, j}^{(p)}$ can be empty. Since $P / \hat{\pi}$ is supposed to be symmetric, we have $\left|V_{i, j}^{(p)}\right|=\left|V_{j, i}^{(p)}\right|$, so that we can define a bijection $\sigma$ between these two sets. Since we can do this for any $i$ and $j$ we can extend the bijection to any of the parts $C_{i}$. If $\sigma(x)=y$ then we put $q(x)=y$ and $q(y)=x$. On the sets $V_{i, i}^{(p)}$ we can choose $\sigma$ to be the identity map. The $q$ we get is a permutation of order 2 on the set of vertices. It is clear that, in this way $\left|V_{i, j}^{(q)}\right|=\left|V_{i, j}^{(p)}\right|=\left|V_{j, i}^{(p)}\right|=\left|V_{j, i}^{(q)}\right|$ and this means that $P / \hat{\pi}=Q / \hat{\pi}$. The statement follows by applying Theorem 20 .

Remark 24. The converse of Corollary 22 is false. In fact, we have seen in Example 13 that, in the case of the cycle $C_{8}$, the partition $\hat{\pi}$ is trivial, so that $P / \hat{\pi}=P$ for any matrix permutation $P$, but we showed that there exist nonsymmetric permutation matrices $P$ such that $A_{C_{8}} P A_{C_{8}}$ is symmetric. Moreover, the converse of Proposition 23 is false, since we showed in Example 13 that there exist nonsymmetric permutation matrices $P$ and symmetric permutation matrices $Q$ such that $A_{C_{8}} P A_{C_{8}}=A_{C_{8}} Q A_{C_{8}}$.

By arguing as in the proof of Proposition 23, we are able to give an estimate of the number of permutations of order 2 giving rise to the same graph in the case in which the matrix $P / \hat{\pi}$ is symmetric. Put $p_{i, j}=\left|V_{i, j}^{(p)}\right|$.

Proposition 25. Let $p_{i, j} / \sqrt{\left|C_{i}\right|\left|C_{j}\right|}$ be the entry $(P / \hat{\pi})_{i, j}$ of the matrix $P / \hat{\pi}$, with $i, j=$ $1, \ldots, m$. There are $\prod_{i, j=1}^{m} p_{i, j}$ ! permutation matrices $Q$ of order 2 such that $\tilde{A}_{H} P \tilde{A}_{H}=$ $\tilde{A}_{H} Q \tilde{A}_{H}$.

Proof. Since the matrix $P / \hat{\pi}$ is symmetric, we have $p_{i, j}=\left|V_{i, j}^{(p)}\right|=\left|V_{j, i}^{(p)}\right|=p_{j, i}$. From the proof of Proposition 23, it is enough to count the number of possible bijections from the set $V_{i, j}^{(p)}$ to $V_{j, i}^{(p)}$ for any $i$ and $j=i, \ldots, m$, i.e., the number of bijections of $V_{i, j}^{(p)}$ to itself. For fixed $i$ and $j$, this number is $p_{i, j} !$. The result follows.

Example 26. Consider the graph $H$ and its permutational 2-nd power in Example 5

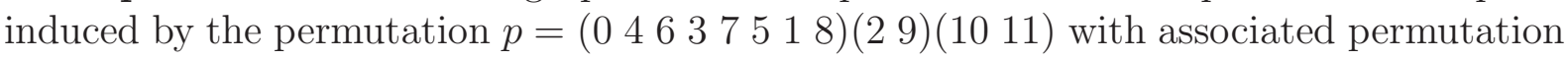
matrix $P$. If $M_{\hat{\pi}}$ is the characteristic matrix of the neighborhood partition on two copies of $H$, one gets:

$$
P / \hat{\pi}=M_{\hat{\pi}}^{T} P M_{\hat{\pi}}=\left(\begin{array}{cccccccc}
0 & 0 & 0 & 1 / 2 & 0 & 1 / \sqrt{2} & 0 & 0 \\
0 & 0 & 0 & 0 & 0 & 0 & 1 & 0 \\
0 & 0 & 0 & 0 & 1 / \sqrt{2} & 0 & 0 & 0 \\
1 / 2 & 0 & 0 & 0 & 1 / 2 & 0 & 0 & 0 \\
0 & 0 & 1 / \sqrt{2} & 1 / 2 & 0 & 0 & 0 & 0 \\
1 / \sqrt{2} & 0 & 0 & 0 & 0 & 0 & 0 & 0 \\
0 & 1 & 0 & 0 & 0 & 0 & 0 & 0 \\
0 & 0 & 0 & 0 & 0 & 0 & 0 & 1
\end{array}\right)
$$

which is a symmetric matrix. By virtue of Proposition 23, there exists a matrix permutation $Q$ of order 2 such that $\left(I_{2} \otimes A_{H}\right) P\left(I_{2} \otimes A_{H}\right)=\left(I_{2} \otimes A_{H}\right) Q\left(I_{2} \otimes A_{H}\right)$. One 
can directly check that a matrix $Q$ of order 2 satisfying this property is the permutation matrix associated with the permutation $q=\left(\begin{array}{ll}0 & 4\end{array}\right)(18)(29)(3$ 7)(5 6)(10 11).

Theorem 27. If $\tilde{A}_{H} / \hat{\pi}$ is invertible and $\tilde{A}_{H} P \tilde{A}_{H}$ is symmetric, then there exists $Q$ of order 2 such that $\tilde{A}_{H} P \tilde{A}_{H}=\tilde{A}_{H} Q \tilde{A}_{H}$.

Proof. If $\tilde{A}_{H} P \tilde{A}_{H}$ is symmetric, then by Corollary 21 the matrix $\left(\tilde{A}_{H} / \hat{\pi}\right)(P / \hat{\pi})\left(\tilde{A}_{H} / \hat{\pi}\right)$ is symmetric; now, since $\tilde{A}_{H} / \hat{\pi}$ is invertible, we deduce that $P / \hat{\pi}$ is symmetric. We can now apply Proposition 23 to get the claim.

Our results are useful when we analyze graphs with an adjacency matrix whose singularity depends on the repetition of some rows. The extreme example is treated in the following section.

\subsection{The complete bipartite graph}

Let $K_{m, n}$ denote the complete bipartite graph on $m+n$ vertices, whose vertex set is partitioned into two sets $V_{1}$ and $V_{2}$, with $\left|V_{1}\right|=m$ and $\left|V_{2}\right|=n$, such that every vertex of $V_{i}$ is connected by an edge to every vertex of $V_{j}$, with $i \neq j$, and no edge connects vertices belonging to the same part. Let $J_{m, n}$ denote the $m \times n$ matrix whose entries are all equal to 1 . Then, up to a reordering of the vertices, the adjacency matrix of $K_{m, n}$ is

$$
A_{K_{m, n}}=\left(\begin{array}{c|c}
O & J_{m, n} \\
\hline J_{n, m} & O
\end{array}\right)
$$

Let us number the vertices of $V_{1}$ by $1,2, \ldots, m$ and the vertices of $V_{2}$ by $m+1, \ldots, m+n$. It is straightforward that the neighborhood partition $\hat{\pi}$ of the vertices of $K_{m, n}$ coincides with the partition $V_{K_{m, n}}=V_{1} \sqcup V_{2}$. In particular, the characteristic matrix $M_{\hat{\pi}}$ is the $(m+n) \times 2$ matrix

$$
M_{\hat{\pi}}=\left(\begin{array}{cc}
1 / \sqrt{m} & 0 \\
\vdots & \vdots \\
1 / \sqrt{m} & 0 \\
0 & 1 / \sqrt{n} \\
\vdots & \vdots \\
0 & 1 / \sqrt{n}
\end{array}\right)
$$

and it satisfies the following equalities:

$$
M_{\hat{\pi}}^{T} M_{\hat{\pi}}=I_{2} ; \quad M_{\hat{\pi}} M_{\hat{\pi}}^{T}=\left(\begin{array}{c|c}
\frac{1}{m} J_{m, m} & 0 \\
\hline 0 & \frac{1}{n} J_{n, n}
\end{array}\right) ; \quad M_{\hat{\pi}}^{T} A_{K_{m, n}} M_{\hat{\pi}}=\left(\begin{array}{cc}
0 & \sqrt{m n} \\
\sqrt{m n} & 0
\end{array}\right) .
$$

The latter matrix can be regarded, up to normalization, as the adjacency matrix of the graph $K_{m, n} / \hat{\pi}$, which reduces to the complete graph on 2 vertices.

Now let $k \geqslant 1$ be a positive integer and consider the disjoint union of $k$ copies of $K_{m, n}$, so that the adjacency matrix of this new graph is given by $\tilde{A}_{K_{m, n}}=I_{k} \otimes A_{K_{m, n}}$, and the 
associated characteristic matrix is $\tilde{M}_{\hat{\pi}}=I_{k} \otimes M_{\hat{\pi}}$. In particular:

$$
\tilde{A}_{K_{m, n}} / \hat{\pi}=\tilde{M}_{\hat{\pi}}^{T} \tilde{A}_{K_{m, n}} \tilde{M}_{\hat{\pi}}=I_{k} \otimes\left(\begin{array}{cc}
0 & \sqrt{m n} \\
\sqrt{m n} & 0
\end{array}\right)
$$

Now let $p$ be a permutation on $k(m+n)$ elements, and let $P$ be the corresponding permutation matrix. As the matrix $\tilde{A}_{K_{m, n}} / \hat{\pi}$ is nonsingular, by virtue of Corollary 21, the matrix $\tilde{A}_{K_{m, n}} P \tilde{A}_{K_{m, n}}$ is symmetric if and only if the matrix $P / \hat{\pi}=\tilde{M}_{\hat{\pi}}^{T} P \tilde{M}_{\hat{\pi}}$ is symmetric. Therefore, by applying Proposition 23, we get the following theorem.

Theorem 28. A permutational $k$-th power of the graph $K_{m, n}$ can always be obtained by a classical zig-zag product. That is, if a permutation matrix $P$ is such that $\tilde{A}_{K_{m, n}} P \tilde{A}_{K_{m, n}}$ is symmetric, then there exists a symmetric permutation matrix $Q$ such that $\tilde{A}_{K_{m, n}} P \tilde{A}_{K_{m, n},}=$ $\tilde{A}_{K_{m, n}} Q \tilde{A}_{K_{m, n}}$.

In order to investigate the symmetry of $P / \hat{\pi}$, observe that $P / \hat{\pi}$ is a square matrix of size $2 k$, whose rows and columns can be indexed by the pairs $(x, y)$, where the copy index $x$ varies in $\{1, \ldots, k\}$ and the part index $y$ varies in $\{1,2\}$. Therefore, one has symmetry if and only if the number of elements in the part $y$ of the copy $x$ which are sent to elements in the part $y^{\prime}$ of the copy $x^{\prime}$ equals the number of elements in the part $y^{\prime}$ of the copy $x^{\prime}$ which are sent to elements in the part $y$ of the copy $x$. Here below an explicit example in the case $k=2, m=3, n=5$.

Example 29. Consider the following permutation of 16 elements

$$
p=\left(\begin{array}{ccc|ccccc|ccc|ccccc}
1 & 2 & 3 & 4 & 5 & 6 & 7 & 8 & 9 & 10 & 11 & 12 & 13 & 14 & 15 & 16 \\
4 & 10 & 16 & 8 & 2 & 9 & 15 & 14 & 6 & 13 & 3 & 7 & 11 & 5 & 12 & 1
\end{array}\right),
$$

with associated permutation matrix $P$. It is easy to check that:

$$
\tilde{M}_{\hat{\pi}}=I_{2} \otimes\left(\begin{array}{cc}
1 / \sqrt{3} & 0 \\
1 / \sqrt{3} & 0 \\
1 / \sqrt{3} & 0 \\
0 & 1 / \sqrt{5} \\
0 & 1 / \sqrt{5} \\
0 & 1 / \sqrt{5} \\
0 & 1 / \sqrt{5} \\
0 & 1 / \sqrt{5}
\end{array}\right) \quad P / \hat{\pi}=\left(\begin{array}{cccc}
0 & 1 / \sqrt{15} & 1 / 3 & 1 / \sqrt{15} \\
1 / \sqrt{15} & 1 / 5 & 1 / \sqrt{15} & 2 / 5 \\
1 / 3 & 1 / \sqrt{15} & 0 & 1 / \sqrt{15} \\
1 / \sqrt{15} & 2 / 5 & 1 / \sqrt{15} & 1 / 5
\end{array}\right)
$$

and therefore the matrix $\left(I_{2} \otimes A_{K_{3,5}}\right) P\left(I_{2} \otimes A_{K_{3,5}}\right)$ is symmetric. A permutation $Q$ of order 2 satisfying the property $\left(I_{2} \otimes A_{K_{3,5}}\right) P\left(I_{2} \otimes A_{K_{3,5}}\right)=\left(I_{2} \otimes A_{K_{3,5}}\right) Q\left(I_{2} \otimes A_{K_{3,5}}\right)$ is $Q=(15)(211)(316)(69)(712)(814)(1013)$, constructed as in the proof of Proposition 23.

\section{Acknowledgements}

The second author thanks the Austrian Science Fund project FWF P29355-N35. 


\section{References}

[1] N. Alon, A. Lubotzky, A. Wigderson, Semi-direct product in groups and zig-zag product in graphs: connections and applications (extended abstract). 42-nd IEEE Symposium on Foundations of Computer Science (Las Vegas, NV, 2001), 630-637. IEEE Computer Soc., Los Alamitos, CA, 2001.

[2] A.E. Brouwer, W.H. Haemers, Spectra of graphs, Universitext, Springer, New York, 2012. xiv +250 pp.

[3] T. Ceccherini-Silberstein, F. Scarabotti, F. Tolli, Discrete harmonic analysis. Representations, number theory, expanders, and the Fourier transform. Cambridge Studies in Advanced Mathematics, 172. Cambridge University Press, Cambridge, 2018. xiii +573 pp.

[4] K.P. Costello, T. Tao, V. Van, Random symmetric matrices are almost surely nonsingular, Duke Math. J. 135 (2006), no. 2, 395-413.

[5] D. D'Angeli, A. Donno, Wreath product of matrices, Linear Algebra Appl. 513 (2017), 276-303.

[6] D. D'Angeli, A. Donno, E. Sava-Huss, Connectedness and isomorphism properties of the zig-zag product of graphs, J. Graph Theory 83 (2016), no. 2, 120-151.

[7] A. Donno, Replacement and zig-zag products, Cayley graphs and Lamplighter random walk, Int. J. Group Theory 2 (2013), no. 1, 11-35.

[8] C.D. Godsil, B.D. McKay, Feasibility conditions for the existence of walk-regular graphs, Linear Algebra Appl. 30 (1980), 51-61.

[9] C. Godsil, Algebraic Graph Theory. Graduate Texts in Mathematics, 207, SpringerVerlag, New York, 2001. xx + 439 pp.

[10] C.D. Godsil, Compact graphs and equitable partitions, Linear Algebra Appl. 255 (1997), 259-266.

[11] R. Hammack, W. Imrich, S. Klavžar, Handbook of product graphs. Second edition. Discrete Mathematics and its Applications (Boca Raton). CRC Press, Boca Raton, $F L, 2011$. xviii +518 pp.

[12] W. Imrich, H. Izbicki, Associative Products of Graphs, Monatsh. Math. 80 (1975), no. 4, 277-281.

[13] A. Lubotzky, Expander graphs in pure and applied mathematics, Bull. Amer. Math. Soc. N.S. 49 (2012), no. 1, 113-162.

[14] H. B. Mann, On linear relations between roots of unity, Mathematika 12 (1965), $107-117$.

[15] O. Reingold, S. Vadhan, A. Wigderson, Entropy Waves, the Zig-Zag Graph Product, and New Constant-Degree Expanders, Ann. of Math. (2) 155 (2002), no. 1, 157-187.

[16] G. Sabidussi, The composition of graphs, Duke Math. J. 26 (1959), 693-696.

[17] A.J. Schwenk, Computing the characteristic polynomial of a graph. Graphs and Combinatorics (Proc. Capital Conf., George Washington Univ., Washington, D.C., 1973), 153-172, Lecture Notes in Math., Vol. 406, Springer, Berlin, 1974. 